S.D. Ryder, D.J. Pisano, M.A. Walker, and K.C. Freeman, eds.

\title{
Cold Molecular Gas as Baryonic Dark Matter
}

\author{
Daniel Pfenniger \\ Geneva Observatory, University of Geneva, Sauverny, Switzerland
}

\begin{abstract}
We review the different cold dark gas models that have been proposed in the literature, as well as a new variant which addresses their principal stability problems by taking into account the property of molecular hydrogen to become solid or liquid below $33 \mathrm{~K}$ and at sufficiently high pressure. This new physical ingredient provides the possibility to stabilise cold gas globules by a core of condensed molecular hydrogen. Such loosely bound cold globules behave in a galaxy as a collisionless ensemble of matter, and form a reservoir of gas easily liberated through, e.g., UV excitation. The cold condensed cores survive the longest, of order a Gyr in the solar neighbourhood radiation field, and much longer in spiral outer HI disks.
\end{abstract}

\section{The Diverse Solutions to the Dark Matter Problems}

While it is customary to speak of the dark matter as if it were a single problem, the history of dark matter, i.e., matter inferred from its gravitational effect but not confirmed otherwise, is a long list of partial and diverse solutions to the general problem. It suffices to recall a few past successful solutions:

- In contrast to the predicted discovery of Neptune (1846) solving outer planets residual motions, which corresponded effectively to finding a new chunk of matter, the predicted Vulcan planet failed (1860-1878) to explain the advance of Mercury's perihelion because the required solution was to update the theory of gravity by general relativity (1916).

- It took time, up to $\sim 1930$, if not up to now in some cases, to appreciate how much light can be absorbed by interstellar dust. Here a truly dark but tiny amount of matter strongly alters the light-estimated mass from a bright matter component.

- The discovery of HI ( $~ 1950)$, CO-inferred $\mathrm{H}_{2}(\gtrsim 1960)$ in spirals, and Xray emitting $\mathrm{H}^{+}$in clusters $(Z 1970)$ did explain a non-negligible fraction of the dynamical mass, without explaining it all. Likewise white and brown dwarf stars solve a part of the dark matter problem.

- It was instructive to learn that, on the one hand, dark matter is strongly suggested especially in late type spirals from the use of the virial theorem applied on the HI rotation curves; while the apparent large but irregular dark matter content in dwarf spheroidals might mostly result from the inappropriate use of the virial theorem on loosely bound stellar systems orbiting in an external field (Kroupa 1997). 
Adopting the Copernican attitude, nowadays there are no grounds to assume that we are at the peculiar epoch at which only a unique solution will solve the remaining dark matter problems. On the contrary, we must expect that dark matter is still a composite problem reflecting the diversity of astrophysical conditions. The recent dark energy evidence just reinforces this view.

Since any mass component possesses several additional properties besides contributing to gravity, ideally a new dark matter component should not only explain the rotation curves, but also its associated physical effects on the galactic machinery. Cold gas is a good case in this respect because it is closely associated with the (also poorly understood) star formation process. Here we concentrate on this baryonic dark matter, discussed in various fashions by several groups (Pfenniger \& Combes 1994 [PC94]; Henriksen \& Widrow 1995; de Paolis et al. 1995; Gerhard \& Silk 1996; Draine 1998; Kalberla \& Kerp 1998; Walker \& Wardle 1998; Sciama 2000a,b; Lawrence 2001). However, we do not forget that excellent arguments exist also to expect substantial amounts of hot intergalactic gas, as well as dominant non-baryonic dark matter(s) at cosmological scales.

\section{Motivations for Expecting Cold Dark $\mathbf{H}_{2}$ in Spirals}

Most of the arguments to expect dark cold gas in spirals presented in Pfenniger, Combes \& Martinet (1994) are still valid. Here are some of them actualised:

- Much of the baryons predicted by the Big Bang nucleosynthesis remain to be found at low redshifts, noting that most of the mass of small scale objects, such as globular clusters, is baryonic. Thus baryons are well separated from non-baryons in the formation process of such objects.

- While dark matter in spirals is correlated to HI (Bosma 1981; Hoekstra et al. 2001), dark matter is anti-correlated to stars along the spiral sequence, as if dark matter transforms into stars (Pfenniger 1996).

- Not only the optical disks are well explained as maximum (Sancisi, this conference), but the HI disks appear dynamically active, as if almost selfgravitating (Kalberla 2003; Masset \& Bureau 2003).

- The ISM gas cools very fast, a few kyr is a typical timescale, while cold $\mathrm{H}_{2}$ and $\mathrm{He}$ below $\approx 500 \mathrm{~K}$ are difficult to observe directly because they do not emit light, and have a tiny sky covering factor in cold virialised states, so easily escape detection by absorption methods.

- The star forming regions in outer galactic disks lacking CO detections also trace the presence of molecules. Perhaps the best evidence for the existence of undetected cold $\mathrm{H}_{2}$ has been provided recently by the remarkable photometric observations of the outer HI disk of M 31 by Cuillandre et al. (2001). The deep photometry of this field reveals the existence of blue stars clearly related to the HI distribution. The almost inescapable consequence is that star formation occurs in situ in a region where no $\mathrm{CO}$ has been detected, so where no molecular gas could be inferred. Star formation means that HI, in conjunction with the detected HI-associated dust, succeeds in condensing there, fragments, and forms dense molecular clumps which finally form stars. The outer HI disk of M 31 appears thus as an active component where gas cycles from a diffuse HI phase to an 
invisible molecular phase. The blue stars formed must photo-dissociate the molecular component, thus opening the possibility to reform HI, and at the same time, to explain the long term existence of HI. HI disks appear as just the visible tips of molecular icebergs.

Of course, the nature of this hidden molecular component must be elucidated.

\section{Cold Dark Gas Models}

Nowadays any viable cold gas model must pass a large set of observational constraints using almost all the electromagnetic spectrum. A model must also pass theoretical constraints, although these are weak in some aspects due to the extraordinary complexity of the ISM. Two types of cold gas models have been proposed in the literature without clear contradictions with observations:

- The spherical cloud models (Henriksen \& Widrow 1995; de Paolis et al. 1995; Gerhard \& Silk 1996; Draine 1998; Kalberla \& Kerp 1998; Walker \& Wardle 1998; Sciama 2000a,b). These spherical cold clouds have sub-pc sizes, belong to a more or less flat pressure supported dark halo, and eventually are clustered and mixed with MACHO's. Their characteristics are not derived from firm physical principles, but constrained by observations. Often these clouds are viewed as long lived pristine clouds from unspecified remote cosmological origins.

The main problem associated with the spherical cloud model is to explain their mere dynamical stability. Indeed isothermal perfect gas clouds without fixed boundaries are known to be unstable with respect to the gravothermal catastrophe on short time-scales, a couple of crossing times. The origin and physics of formation of these clouds is an open question.

- The fractal gas model (PC94; Combes \& Pfenniger 1997). This model is mainly an extension of the observed fractal distribution adopted by the CO-bright molecular clouds from kpc to sub-mpc scale. The invisible molecular component is here associated with the HI outer disk. The smallest scales units ("clumpuscules") are determined by the opacity limited fragmentation $\left(\tau_{\text {dyn }}=\tau_{\text {Kelvin-Helmoltz }}\right.$ ) (Low \& Lynden-Bell 1976; Rees 1976): they have Jupiter mass and solar-system size at a temperature of $T \gtrsim 3 \mathrm{~K}$. The fractal has a disk distribution associated to HI, which is the diffuse phase of the fractal gas. As a whole, the fractal is a dynamical structure in which fragmentation and dissolution occur constantly without much star formation.

The main problem with the fractal gas model is explaining its long term dynamical stability, at large as well as small scales. Also the long term dynamical stability of a massive galactic disk composed of such a fractal massive gas distribution requires a sufficiently high effective velocity dispersion, but this quantity is bounded by the largest fractal scale, the one of the diffuse gas. If the HI velocity dispersion is a measure of the fractal largest velocity dispersion, then it is too low by a factor $2-7$ depending on the galaxy.

So both types of models have attractive features, but also weaknesses. 


\section{A Revised Cold Dark Gas Model}

\subsection{Outline of the Model}

Now we describe in broad terms a revised fractal model including globules (Pfenniger 2004), which is an attempt to address the difficulties of both types of cold gas model while keeping their attractive properties.

Here we drop the assumption that the clumpuscules mostly succeed in avoiding collapse. But in low temperature and low opacity conditions, collapse leads to the formation of cold globules stabilised in the core by solid/liquid $\mathrm{H}_{2}$, instead of stars. Stars form in decreasing proportion as ambient heating and ISM FIR opacity decrease. The cold globules evaporate as a function of ambient heating, but can be Gyr long-lived in cold, low-excitation conditions. Over time the globules diffuse away from the disk as stars do (Wielen's diffusion) and by increasing their velocity dispersion contribute to marginally stabilising the disk.

\subsection{Gravitational Collapse and Opacity}

Central to the discussion of the cold gas fate is the outcome of gravitational collapse, because collapse is usually associated with formation of hot bodies such as stars or planets. Below we show how the opposite outcome, bodies based on a cold core, is another possibility.

In classical spherical collapse studies (e.g., Larson 1969), one learns that a spherical collapse of an initial cold $(T \approx 10 \mathrm{~K})$ ISM gas cloud increases its central density almost isothermally towards infinity in a finite time, a singular event. Isothermality results from the large transparency of the cold ISM to its own thermally generated photons, such that the cooling time remains much shorter than the free-fall time. However in the growing density collapsing core, an optical depth exceeding unity is finally reached, allowing the core to keep thermal photons longer, which leads to increasing central temperature and pressure, stopping the singular density growth. All this supposes the gas equation of state remains perfect all the way.

Let us estimate the central density at which the center of a pseudo isothermal sphere

$$
\rho(r)=\frac{\rho_{0}}{1+\left(r^{2} / r_{c}^{2}\right)}, \quad M(r)=4 \pi \rho_{0} r_{c}^{3}\left[\frac{r}{r_{c}}-\arctan \left(\frac{r}{r_{c}}\right)\right],
$$

becomes opaque for constant opacity $\kappa$. The optical depth from the center to infinity is:

$$
\tau_{\infty}=\int_{0}^{\infty} \kappa \rho(r) d r=\frac{\pi}{2} \kappa \rho_{0} r_{c} .
$$

Requiring that $\tau_{\infty}=1$ and that the pseudo isothermal sphere is in dynamical equilibrium,

$$
\lim _{r \rightarrow \infty} \frac{G M(r)}{r}=\frac{3 k T}{\mu m_{p}},
$$

leads to a simple expression for the core radius and the critical central density above which the center begins to be opaque:

$$
r_{c}=\frac{3 \kappa k T}{8 G \mu m_{p}}, \quad \rho_{0}=\frac{16 G \mu m_{p}}{3 \pi \kappa^{2} k T} \approx 2.7 \times 10^{-8} \frac{(\mu / 2)}{\left(\kappa / 10^{-4}\right)^{2}(T / 10)}\left[\mathrm{g} \mathrm{cm}^{-3}\right] .
$$






Figure 1. $\mathrm{H}_{2}$ equation of state in the range $1<T<100 \mathrm{~K}$ and $10^{-43}<P<10^{3}$ bar (Pfenniger 2004)

This critical density is independent of the mass or size of the cloud. Now, for general equations of state the temperature increase in a homologous collapse is related to the density increase (see, e.g., Padmanabhan 2001 [Fig. 3.7]):

$$
\frac{d T}{T}=\left(\frac{4 \alpha-3}{3 \delta}\right) \frac{d \rho}{\rho}, \quad \text { where } \quad \alpha \equiv\left(\frac{\partial \ln \rho}{\partial \ln P}\right)_{T}, \quad \delta \equiv-\left(\frac{\partial \ln \rho}{\partial \ln T}\right)_{P} .
$$

So when the isothermal compressibility $\alpha$ decreases below $3 / 4$, further compression leads to cooling, a reversal of the usual perfect gas behavior. In normal stellar or planetary conditions compressibility decreases when matter is subject to electron degeneracy, but the same effect holds for any hardly compressible matter: it cools down when compressed.

\subsection{Liquid or Solid $\mathbf{H}_{2}$}

Molecular hydrogen, the bulk of cold gas, becomes liquid or solid, i.e., almost incompressible, below $33 \mathrm{~K}$ at sufficient pressure. The $\mathrm{H}_{2}$ critical point occurs at $T_{c}=33 \mathrm{~K}, P_{c}=13 \mathrm{bar}$, while the triple point is at $T_{3}=14 \mathrm{~K}, P_{3}=0.07 \mathrm{bar}$. At low $T$, the saturation pressure and gas density drops to interstellar values. Between 1 and $14 \mathrm{~K}$ the sublimation curve is well approximated by (PC94):

$$
P_{\text {solid-gas }} \approx 5.7 \cdot 10^{20} T^{5 / 2} \mathrm{e}^{-91.75 / T}\left[\mathrm{~K} \mathrm{~cm}^{-3}\right] .
$$


An analytical expression for the full $\mathrm{H}_{2}$ equation of state $\rho(P, T)$ in cold conditions has been derived in the temperature range $[1-100] \mathrm{K}$, from cosmic pressures up to $1 \mathrm{kbar}$, by gathering laboratory data from the literature (Pfenniger 2004). Fig. 1 shows the analytical fit to the indicated data points displaying the steep density wall that occurs below $33 \mathrm{~K}$, breaking the perfect gas inclined plane.

The $\mathrm{H}_{2}$ gas density $\rho$ at the critical pressure is almost the perfect gas density for the partial pressure $P_{\text {solid-gas }}$. Equating this density $\rho$ to the critically opaque density $\rho_{0}$ above provides the critical opacity $\kappa_{\text {crit }}$ below which $\mathrm{H}_{2}$ becomes incompressible before becoming opaque to its thermal radiation:

$$
\kappa_{\text {crit }} \approx 1.3 \times 10^{-3}(T / 5)^{-5 / 4} \mathrm{e}^{9.18 /(T / 5)}\left[\mathrm{cm}^{2} \mathrm{~g}^{-1}\right]
$$

Since the ISM sub-mm opacities typical of low metallicity cold clouds can be as low as $\sim 10^{-3}\left[\mathrm{~cm}^{2} / \mathrm{g}\right.$ ] or less (see Draine 2004, [Fig. 12]), at least below $\sim 5 \mathrm{~K}$ a collapsing cloud is able to condense $\mathrm{H}_{2}$ in the core before becoming opaque. Further compression of the condensed core, instead of heating it, cools it. The condensed core is thus able to stop collapsing without becoming hot. Somewhat warmer or more opaque initial gas can still reach $\mathrm{H}_{2}$ condensation despite a temporary temperature increase, provided the core remains below $33 \mathrm{~K}$.

We arrive thus at the surprising possibility that gravitational collapse in cold conditions leads not necessarily to a body with a hot center, such as planets or stars, but to a temperature inverted gas globule with a condensed cold core. The core is made of solid $\mathrm{H}_{2}$ below $14 \mathrm{~K}$, or liquid $\mathrm{H}_{2}$ above, up to $33 \mathrm{~K}$. Around the core an $\mathrm{H}_{2}$-He gaseous atmosphere may extend to distances much larger than the core size, and thus makes most of the globule mass.

The typical properties of such cold gas condensations have been calculated by integrating the equilibrium equations for isothermal gravitating spheres for which the accurate $\mathrm{H}_{2}$ equation of state is used (Pfenniger 2004). Summarising, the main determined properties of the condensed $\mathrm{H}_{2}$ based globule models are:

a) The central density is determined by the solid or liquid $\mathrm{H}_{2}$ density: $0.07-$ $0.09 \mathrm{~g} \mathrm{~cm}^{-3}$. This is much lower, by a factor $20-50$, than for normal planets.

b) The maximum core radius is about $2 \times 10^{4} \mathrm{~km}$, which corresponds to a maximum core mass similar to the Earth's mass (this is the analog of Chandrasekhar's mass for white dwarfs). At this maximum radius the gravitational and van der Waals binding energies are similar.

c) The cold globules have a relatively weak gravitational binding energy: about $0.001 \mathrm{eV} /$ nucleon, which means that such globules can be rapidly evaporated by sufficient radiative or cosmic ray excitation, but the most dense parts, especially the cores, can survive several Gyr in low excitation regions. In other words, repeated cycles of collapse/evaporation trap an increasing fraction of the gas mass in condensed cores.

d) An extended atmosphere, extending up to 3-30 AU for a Jupiter mass, has an isothermal profile $\propto r^{-2}$, independent of the central core pressure. The atmosphere is expected to be transparent to sub-mm/FIR radiation, but opaque to visible/UV radiation (Combes \& Pfenniger 1997).

It should be noted that the external "heating" of cold globules by penetrating radiation (FIR, cosmic rays) does not increase their apparent temperature due to the negative specific capacity of self-gravitating objects. Such "heating" 
expands and evaporates the atmosphere without increasing temperature, i.e., without a particular emission signature.

\subsection{Observational Counterparts}

The best present observational candidates for cold globules are the cometary knots directly observed in nearby planetary nebulae such as in the Helix Nebula (NGC 7293), but not satisfactorily explained (e.g., O'Dell et al. 2002). Their sizes $(\approx 100 \mathrm{AU})$, their temperature $(\lesssim 5 \mathrm{~K})$, and their centrally concentrated profiles (Burkert \& O'Dell 1998) indicate that they are gravitationally bound around a dense center, not pressure confined clouds. The photo-evaporation action of the central star makes them visible, and their outward motion indicates that they formed in a few kyr from the expanding and cooling gas shells. They illustrate how fast cosmic gas cools and produces clumps; the condensation process leading to such gravitationally bound clouds must occur even more frequently and generally in less heated conditions of the ISM, such as in the outer galactic disks.

Less direct evidence for AU-sized condensations are provided by the Extreme Scattering Events (ESE's) (see PC94; Walker \& Wardle 1998), which are anomalous radio scintillations of quasars or pulsars lasting weeks caused by localised concentrations of electrons. The inferred high electron densities are best explained from the residual ionisation produced by cosmic rays and UV radiation in dense clouds of almost neutral and cold gas.

Lawrence (2001) argued that a part of the unidentified SCUBA sources might be compatible with a population of Galactic cold clouds with properties compatible with the clouds discussed here.

\section{Conclusions}

A substantial amount of cold gas provides not only an explanation for the composition of spirals, especially late types, but also how they process their gas into stars. Cold gas globules stabilised by solid/liquid $\mathrm{H}_{2}$ cores may constitute the cold side of the star formation process. The effect is general and explains the inefficiency of normal star formation. Collapse does not need to be prevented; stars should form in higher fractions in more radiation excited, or more metal enriched (opaque) conditions, and conversely for cold globules. The anticorrelation of dark matter and stars in spirals is then natural, as well as the association of $\mathrm{HI}$ and some star formation in outer galactic disks.

The ISM fast cooling problem is removed if the outcome of collapse can be cold globules instead of stars: the ISM appears then as a very active medium constantly recycling gas from large to small scales. Cold globules are in some way the IMF low mass end. Their low binding energy allows them to play the role of a gas reservoir easily accessible via excitation. As the globule evaporation timescale is inversely proportional to radius, at different galactic radii an equilibrium distribution of globule sizes can be reached balancing the local excitation and gas cooling rates. According to galactic N-body models (Revaz \& Pfenniger 2004) over most of the galaxy evolution the fraction of cold gas remains large with respect to visible gas, thus playing the role of a dark component. 
In outer gaseous disks the densest parts of cold globules should survive over Gyr and as a collisionless population, should play the stabilising role that stars have in the optical disks due to their increasing velocity dispersion. Thus massive self-gravitating stable disks are possible with effective thickness not much larger than the visible HI layer (see Kalberla 2003).

Acknowledgments. This work was supported by the Swiss National Science Foundation.

\section{References}

Bosma, A. 1981, AJ, 86, 1791

Burkert, A., \& O'Dell, C.R. 1998, ApJ, 503, 792

Combes, F., \& Pfenniger, D. 1997, A\&A, 327, 453

Cuillandre, J.-Ch., et al. 2001, ApJ, 554, 190

de Paolis, F., Ingrosso, G., Jetzer, P., \& Roncadelli, M. 1995, A\&A, 295, 567

Draine, B.T. 1998, ApJ, 509, L41

Draine, B.T. 2004, in "The Cold Universe", Saas Fee Advanced Course 32, D. Pfenniger \& Y. Revaz (eds.), Springer, Berlin, p. 213

Gerhard, O., \& Silk, J. 1996, ApJ, 472, 34

Henriksen, R.N., \& Widrow, L.M. 1995, A\&A, 441, 70

Hoekstra, H., van Albada, T.S., \& Sancisi, R. 2001, MNRAS, 323, 453

Kalberla, P.M.W. 2003, ApJ, 588, 805

Kalberla, P.M.W., \& Kerp, J. 1998, A\&A, 339, 745

Kroupa, P. 1997, New Astron., 2, 139

Larson, R.B. 1969, MNRAS, 145, 271

Lawrence, A. 2001, MNRAS, 323, 147

Low, C., \& Lynden-Bell, D. 1976, MNRAS, 176, 367

Masset, F.S., \& Bureau, M. 2003, ApJ, 586, 152

O'Dell, C.R., et al. 2002, AJ, 123, 3329

Padmanabhan, T. 2001, Theoretical Astrophysics Vol. II, Cambridge

Pfenniger, D. 1996, in "Barred Galaxies and Circumstellar Activity", Nobel Symp. 98, Aa. Sandqvist \& P.O. Lindblad (eds.), Springer, Berlin, p. 91

Pfenniger, D. 2004, A\&A, to be submitted

Pfenniger D., \& Combes F. 1994, A\&A, 285, 93 (PC94)

Pfenniger D., Combes F., \& Martinet L. 1994, A\&A, 285, 79

Rees, M.J. 1976, MNRAS, 176, 483

Revaz, Y., \& Pfenniger, D. 2004, A\&A, to be submitted

Sciama, D.W. 2000a, MNRAS, 312, 33

Sciama, D.W. 2000b, MNRAS, 319, 1001

Walker, M., \& Wardle, M. 1998, ApJ, 498, L125 